\title{
Cyclodextrin-based photoactive liposomal nanoparticles for tumor targeting
}

Ilya Yakavets, Henri-Pierre Lassalle, Vladimir Zorin, Lina Bezdetnaya

Ilya Yakavets, Henri-Pierre Lassalle, Vladimir Zorin, Lina Bezdetnaya, "Cyclodextrin-based photoactive liposomal nanoparticles for tumor targeting," Proc. SPIE 11070, 17th International Photodynamic Association World Congress, 110701P (7 August 2019); doi: 10.1117/12.2524210

Event: 17th International Photodynamic Association World Congress, 2019, Cambridge, Massachusetts, United States 


\title{
Cyclodextrin-based photoactive liposomal nanoparticles for tumor targeting
}

\author{
Ilya Yakavets*,a,b,c, Henri-Pierre Lassalle ${ }^{\mathrm{a}, \mathrm{b}}$, Vladimir Zorin ${ }^{\mathrm{c}, \mathrm{d}}$, Lina Bezdetnaya ${ }^{\mathrm{a}, \mathrm{b}}$ \\ ${ }^{a}$ Centre de Recherche en Automatique de Nancy, Centre National de la Recherche Scientifique \\ UMR 7039, Université de Lorraine, Campus Sciences, Boulevard des Aiguillette, 54506 \\ Vandoeuvre-lès-Nancy, France; ${ }^{b}$ Research Department, Institut de Cancérologie de Lorraine, 6 \\ avenue de Bourgogne, 54519 Vandoeuvre-lès-Nancy, France ; ' Laboratory of Biophysics and \\ Biotechnology, Belarusian State University, 4 Nezavisimosti Avenue, 220030 Minsk, Belarus; \\ d International Sakharov Environmental Institute, Belarusian State University, Dauhabrodskaja 23, \\ 220030 Minsk, Belarus
}

\begin{abstract}
The present study is aimed at the development of drug-in-cyclodextrin-in-liposome (DCL) nanoconstruct by coupling two independent delivery systems: cyclodextrin/mTHPC inclusion complexes and liposomal vesicles to improve the transport of mTHPC to the target tissue and to strengthen its intra-tissue accumulation in the tumor. Liposomes offer an excellent opportunity to achieve selective drug, targeting what is expected to prevent local irritation and reduce drug toxicity. Cyclodextrins (CDs) have been utilized as independent carriers for improvement of pharmaceutical properties such as solubility, stability, and bioavailability of various drug molecules, including mTHPC. Therefore, we assumed that encapsulation of CD-complexed drug into liposomes might increase drug loading capacity, entrapment efficiency, may restrain the dissociation of drug-CD complexes and prolong its systemic circulation.

DCL nanoparticles have been prepared with various compositions to optimize the structure aiming to alter more favorably the distribution of temoporfin in tumor tissue. To enhance the encapsulation efficiency, double loaded DCLs, which include mTHPC in lipid bilayer along with (CD-mTHPC) inclusion complexes in the inner aqueous lumen, were prepared. It was demonstrated that DCLs possessed higher serum stability compared with conventional mTHPC liposomes $\left(\right.$ Foslip $\left.^{\circledR}\right)$. In fine, we showed that the presence of serum in the medium less affected cellular uptake of mTHPC delivered by double loaded MDCL compared with Foslip ${ }^{\circledR}$.
\end{abstract}

Keywords: PDT, temoporfin, cyclodextrins, liposomes, serum.

\section{INTRODUCTION}

Photodynamic therapy is a minimally invasive an FDA-approved anticancer modality with a promising clinical track record for oncological and other diseases. The action of PDT consists in the interaction of three principal components: (i) a light-absorbing compound that initiates a photochemical or photophysical reaction, namely photosensitizer (PS), (ii) the light of a specific wavelength and (iii) molecular oxygen ${ }^{1}$. The interaction between these components leads to the generation of reactive oxygen species (ROS), which, due to their toxicity, could damage tumor tissue by direct and indirect way ${ }^{2,3}$. PDT is considered as a highly selective due to the irradiation of the particular target site and the selective uptake of PS in the malignant tissues. To date, the investigations in PDT relate to the application of nanomaterial-based drug delivery systems to increase the selectivity of tumor targeting and overcome the water insolubility of certain PSs ${ }^{4}$.

The present work deals with temoporfin (meta-tetra(3-hydroxyphenyl)chlorin, mTHPC), a highly potent secondgeneration PS. In 2001, the commercial medicinal product based on mTHPC (Foscan ${ }^{\circledR}$ ) had been already approved by the European Medicines Agency for the palliative treatment of advanced head and neck squamous cell carcinoma ${ }^{5,6}$. mTHPC possesses pure-compound preparation, hence efficient light tissue penetration, specific selectivity of tumor uptake, attractive photophysical characteristics and requires low light doses and concentrations to be photoactive ${ }^{7}$. To date, numerous nanoparticles were applied for mTHPC delivery ${ }^{8}$. Nonetheless, the majority of used nanomaterials for mTHPC delivery have drawbacks, such as the quick uncontrolled release of the drug, rapid clearance, limited penetration in tissues. Recently, the hybrid nanoplatforms as a combination of nanoparticles have been suggested as an advanced

17th International Photodynamic Association World Congress, edited by Tayyaba Hasan, Proc. of SPIE Vol. 11070, 110701P · C 2019 SPIE · CCC code: 0277-786X/19/\$21 - doi: 10.1117/12.2524210 
alternative strategy for mTHPC delivery ${ }^{8,9}$. The present work is a continuation of the study of a hybrid delivery system based on the combination of liposomes and cyclodextrins for temoporfin.

Recently, we reported on the development of Drug-in-Cyclodextrin-in-Liposome (DCL) formulation of mTHPC (Figure 1) ${ }^{9}$. Such hybrid nanoconstruct was firstly proposed by McCormack and Gregoriadis (1994) ${ }^{10}$, and has been already applied to encapsulate a variety of lipophilic drugs including anesthetic, immunosuppressive, anti-inflammatory, and anti-cancer drugs ${ }^{11,12}$. In our recent report ${ }^{9}$, we demonstrated the possibility to combine the advantages of liposomal and inclusion complex-based delivery systems in mTHPC-DCLs. Cyclodextrins (CDs) have been utilized as an independent carrier for mTHPC, improving its accumulation and penetration in tumor tissue models ${ }^{13,14}$, while liposomes, as drug containers, offer an excellent opportunity for selective delivery of mTHPC to the tumor tissues ${ }^{15}$. Thus, we suggested that the coupling of both delivery systems in DCL could restrain the dissociation of drug-mTHPC complexes, avoid rapid drug release, and contribute to the altering PSs pharmacokinetics in vivo. With this aim, we compared the behavior ofsingle and double mTHPC-loaded DCLs and conventional liposomal formulation of mTHPC $\left(\right.$ Foslip $^{\circledR}$ ) in the presence of serum proteins.
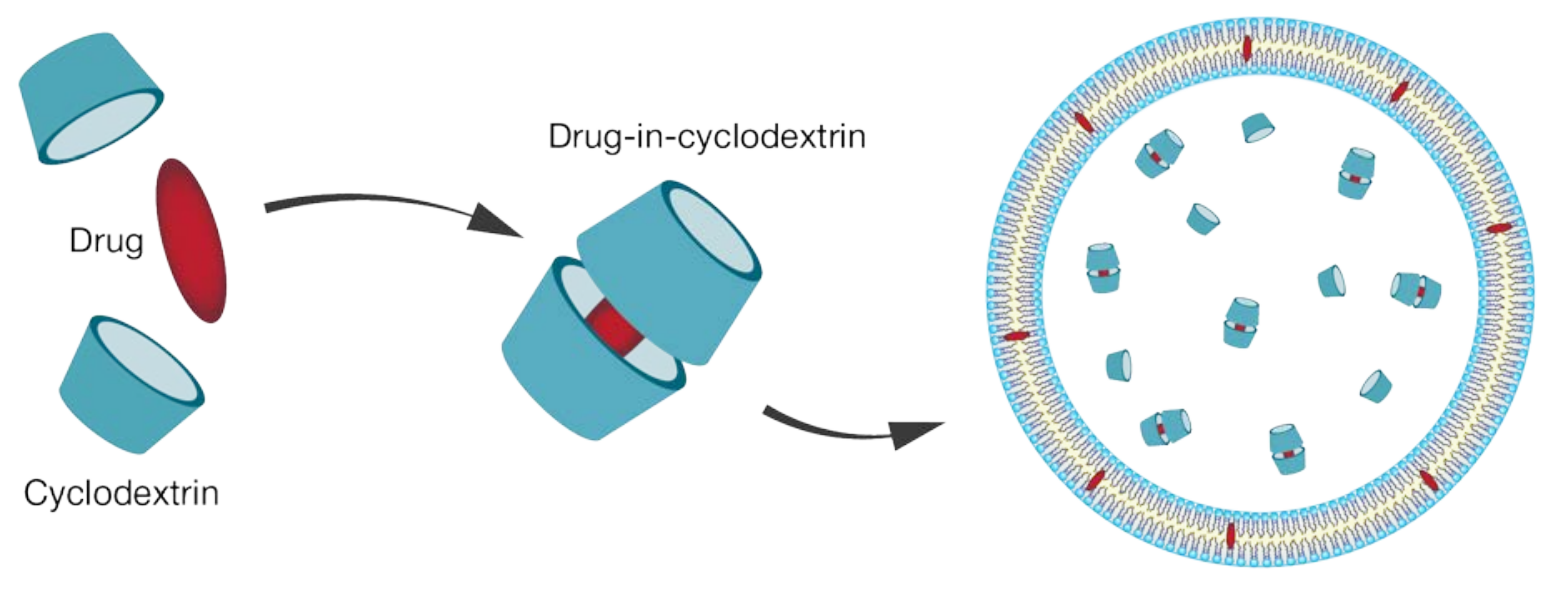

Drug-in-cyclodextrin-in-liposome

Figure 1. The scheme of Drug-in-Cyclodextrin-in-Liposome formulation

\section{MATERIALS AND METHODS}

\subsection{Materials}

mTHPC stock solution (10 mM) was prepared in methanol and kept at $4{ }^{\circ} \mathrm{C}$ in the dark. The concentration of mTHPC in the solution was estimated spectroscopically using molar extinction coefficient of $30,000 \mathrm{M}^{-1} \mathrm{~cm}^{-1}$ at $650 \mathrm{~nm}$ in ethanol ${ }^{16}$. L-a-dipalmitoylphosphatidyl- choline (DPPC) and dipalmitoylphosphatidylglycerol (DPPG) were purchased from (Sigma, USA). Random methyl- $\beta$-cyclodextrin (Me- $\beta-C D)$ was purchased from CYCLOLAB R\&D. Ltd., (Budapest, Hungary). Human blood serum was collected from healthy donors according to the protocol previously described ${ }^{17}$.

\subsection{Liposomal vesicles preparation}

DCLs were prepared by thin lipid film hydration method, as was previously described ${ }^{9}$. Briefly, Inclusion complexes of mTHPC with $\beta$-CDs were formed using solvent co-evaporation method in ultrapure water (Millipore, Milli- $Q^{\circledR}$ Advantage A10 ${ }^{\circledR}$ System, Millipore, Eschborn, Germany). Unilamellar liposomes contained a combination of DPPC and DPPG at a molar ratio 9:1 and were made by membrane extrusion technique with a final concentration of lipid $15 \mathrm{mg} / \mathrm{mL}$. In the case of double-loaded DCLs, mTHPC were added at the step of the preparation of lipid mixture at a molar drug:lipid ratio 1:20. The encapsulation of inclusion complexes was performed at the lipid film hydration step. The purification of DCLs from the not encapsulated mTHPC/ $\beta$-CDs in the medium was performed using a minicolumn chromatography technique ${ }^{18}$. 
Liposomal mTHPC formulation, Foslip ${ }^{\circledR}$, was kindly provided by biolitec research GmbH (Jena, Germany). Foslip ${ }^{\circledR}$ was reconstituted from lyophilized powder in distilled water as per the manufacturer's instructions.

\subsection{Characterization}

The hydrodynamic diameter of nanoparticles, polydispersity index (PDI), and surface charge were determined using dynamic light scattering technique by Zetasizer Nano ZS (Malvern Instruments, UK). The samples were diluted in ultrapure water at $25^{\circ} \mathrm{C}$ and measured at the angle of $173^{\circ}$. The hydrodynamic diameter was estimated from the autocorrelation function of the intensity scattered from the particles light, assuming a spherical particle shape, a medium viscosity of $0.89 \mathrm{mPas}$ and a refractive index of 1.33 . To estimate the encapsulation efficiency of mTHPC in DCLs, we measured the optical density of samples at $652 \mathrm{~nm}$ immediately after extrusion $\left(\mathrm{A}_{\text {total }}\right)$ and purification $\left(\mathrm{A}_{\text {final }}\right)$. Before measurements, the samples diluted 1500 times in $0.2 \%$ Triton $^{\circledR} \mathrm{X}-100$ solution. The value of encapsulation efficiency was estimated as $\mathrm{A}_{\text {final }} / \mathrm{A}_{\text {total }} \times 100 \%$.

\subsection{Size-exclusion chromatography}

Chromatographic experiments were performed on a Sigma $1.5 \times 75 \mathrm{~cm}$ column filled with Sepharose CL-6B gel (GE Healthcare, USA) pre-equilibrated with PBS with total bed volume $150 \mathrm{~mL}$. mTHPC nanoformulations $(5 \mu \mathrm{M})$ were incubated for $24 \mathrm{~h}$ in PBS supplemented with $50 \%$ of human serum at $37^{\circ} \mathrm{C}$. After incubation, the sample was injected into the column using a three-way connector. The sample volume was $1.0 \mathrm{~mL}$, and flow speed was set to $1 \mathrm{~mL}$ per min. Fractions of $1 \mathrm{~mL}$ were collected by an automated fraction collector. The column was stored at room temperature, and the separation was carried out in a partially light- protected environment to avoid mTHPC photobleaching effects. Fractions with elution volume from 25 to $121 \mathrm{~mL}$ were collected and analyzed for mTHPC content using SAFAS Xenius XM (SAFAS, Monaco, France) spectrofluorometer. mTHPC content in the chromatographic fractions was estimated by fluorescence intensity measured after the addition of $0.2 \%$ Triton ${ }^{\circledR}$ X-100 (Sigma-Aldrich) to the samples. The decomposition of elution profiles on Gauss bands was performed using Origin software (OriginLab, Northampton, MA, USA).

\subsection{Cell culture}

For in vitro cell culture experiments, we used HT29 human adenocarcinoma cells, obtained from the ATCC ${ }^{\circledR}$ (LGC Promochem, Molsheim, France). The cells were maintained in phenol red-free Roswell Park Memorial Institute 1640 medium (RPMI-1640, Invitrogen ${ }^{\mathrm{TM}}$, Carlsbad, California, USA) medium supplemented with $9 \%$ (vol/vol) heatinactivated fetal bovine serum (FBS) (Life Technologies, Carlsbad, California, USA), penicillin (10,000 IU), streptomycin $(10,000 \mu \mathrm{g} / \mathrm{mL}$ ), and $1 \%$ (vol/vol) $0.1 \mathrm{M}$ glutamine (Life Technologies, Carlsbad, CA, USA). Cells were kept as a monolayer culture in a humidified incubator $\left(5 \% \mathrm{CO}_{2}\right)$ at $37^{\circ} \mathrm{C}$. Monolayer cell culture was reseeded every week to ensure exponential growth and regularly controlled for mycoplasma contamination.

\subsection{Flow cytometry}

The HT29 monolayer cells were incubated with mTHPC nanoformulations (1.5 $\mu \mathrm{M})$ for 24h in RPMI-1640 supplemented with various serum concentrations. Cells were trypsinized, washed two times with buffer and obtained suspension was analyzed using FACSCalibur (BD, Franklin Lakes, USA), equipped with a laser emitting at $633 \mathrm{~nm}$. The mTHPC fluorescence was detected in fluorescence channel FL4 with a $661 \pm 16 \mathrm{~nm}$ filter.

\subsection{Fluorescence microscopy}

mTHPC fluorescence was observed under an upright epifluorescence microscope (AX-70 Provis, Olympus, France) equipped with a $100 \mathrm{~W}$ mercury vapor lamp and a Peltier cooled CCD camera (DP70, Olympus). The filter was set at 400-440 nm bandpass excitation associated with a $570 \mathrm{~nm}$ dichroic mirror and a $590 \mathrm{~nm}$ long pass emission filter for mTHPC fluorescence measurements. HT29 cells $\left(3 \times 10^{4} \mathrm{cells} / \mathrm{ml}\right)$ were plated into Lab-Tek II chamber Slide (Roskilde, Denmark), incubated in the dark at $37^{\circ} \mathrm{C}$ with $4.5 \mu \mathrm{M}$ of mTHPC in different formulations for 3 hours and then rinsed with PBS. Photosensitizer incubation was performed in the dark at $37^{\circ} \mathrm{C}$ in a humidified $5 \% \mathrm{CO}_{2}$ air atmosphere for $24 \mathrm{~h}$. Fluorescence images were recorded using an oil immersion $\times 40$ objective in the case of monolayer cell culture.

\subsection{Statistics}

Statistical analysis was carried out using the Student's t-test. Statistical significance was established at $P<0.05$. All experiments were repeated at least three times, and results are expressed as mean values \pm S.D. 


\section{RESULTS AND DISCUSSIONS}

\subsection{Characterization}

We synthesized two types of DCLs loaded with mTHPC/Me- $\beta-C D$ inclusion complexes. The single loaded nanoconstruct (MDCL) encapsulated mTHPC/Me- $\beta$-CD inclusion complexes only in the inner aqueous core of liposomal vesicles, while the double loaded (MDCL DL) contained mTHPC in lipid bilayer as well as in inclusion complexes inside of liposomes. The hydrodynamic diameters of MDCL and MDCL DL were measured using dynamic light scattering and were $133.2 \pm 0.8 \mathrm{~nm}$ and $143.2 \pm 1.5 \mathrm{~nm}$, respectively. Both samples have a narrow size distribution with PDI $<0.06$. The lipid membrane for both nanoconstructs consists of DPPC and DPPG lipids in the molar ratio of 9:1 determining the negative surface charge of vesicles about $(-35)-(-38) \mathrm{mV}$. The colloidal stability of DCLs was observed for 3 months. The encapsulation efficiency of mTHPC in MDCL and MDCL DL was 5\% and $11 \%$. The presence of mTHPCin the soluble form of inclusion complexes was confirmed by spectroscopic techniques described previously ${ }^{9,19,20}$.

\subsection{Stability in serum}

Earlier, the rapid destruction of conventional liposomes loaded with mTHPC (Foslip ${ }^{\circledR}$ ) in the presence of plasma proteins was reported ${ }^{17,21}$. To assess the structural stability of DCLs in human plasma, we used size-exclusion chromatography technique. Size-exclusion chromatography is the traditional method to analyze the affinity of the pharmaceutical compounds for serum proteins based on the separation of the serum into individual protein fractions according to their size ${ }^{17,19}$. The large-scale components don't pass to the gel beads, so they are excluded first, while small components pass through the gel beads and are excluded the last. The obtained fractions could be analyzed by fluorimetric technique to estimate the amount of PS in each fraction.

Separation of serum samples contained mTHPC nanoformulations (Foslip ${ }^{\circledR}$, MDCL, and MDCL DL) allows estimation of PS distribution between the serum components (Figure 2). After 24h of incubation of Foslip ${ }^{\circledR}$ with $50 \%$ human serum, mTHPC was eluted in three distinct bands with maxima at 45, 60 and $78 \mathrm{~mL}$ (Figure 2), corresponding to liposomes, low-density lipoproteins (LDL) and high-density lipoproteins (HDL). There was only a small peak at $45 \mathrm{~mL}$ related to the mTHPC remained in Foslip ${ }^{\circledR} 24 \mathrm{~h}$ post-incubation. The data obtained correlates with the previously published results ${ }^{17,21}$. The stability of liposomes is affected by serum proteins which bind to the vesicle's surface promoting the transfer of membrane components ${ }^{22}$. That leads to the fragmentation of liposomal membrane and redistribution of membrane components, including PS, to the serum proteins ${ }^{21}$.

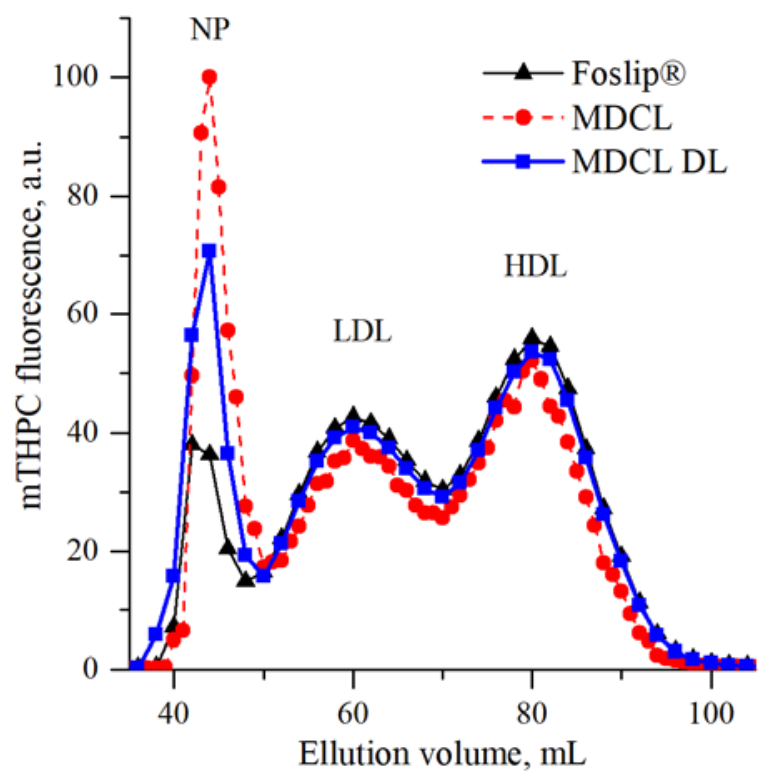

Figure 2. Elution profiles of mTHPC from the different formulation in the presence of $50 \%$ human serum. Incubation time was $24 \mathrm{~h}$. The concentration of mTHPC was $5 \mu \mathrm{M}$. 
In the case of DCLs, the measured distribution profiles of mTHPC after 24h incubation in 50\% human serum also exhibited three distinct bands. However, the intensities of these peaks were significantly different compared to Foslip ${ }^{\circledR}$. Indeed, the peak corresponded to NPs was considerably higher in the case of DCLs compared to Foslip ${ }^{\circledR}$, displaying a higher amount of mTHPC remained in hybrid nanoconstructs. At the same time, protein-bound mTHPC concentration slightly decreased compared with Foslip ${ }^{\circledR}$.

Comparing mTHPC content in liposome- and protein-based bands, we calculate the relative fraction of mTHPC in each band and using decomposition analysis. The estimated quantitative distribution of mTHPC among serum proteins and liposomal vesicles is presented in Figure 3. According to the data obtained, the release of mTHPC from Foslip ${ }^{\circledR}$ reached $88 \%$, while only $12 \%$ of PS was bound to liposomes after 24h of incubation. On the other hand, DCL seems to be more stable in serum, hence the percentage of mTHPC in MDCL and MDCL DL was 22\% and 18\% respectively. Earlier, the increased serum stability of DCL formulation was reported by Chen et al. (2007) ${ }^{23}$ and Maestrelli et al. (2006) ${ }^{24}$.

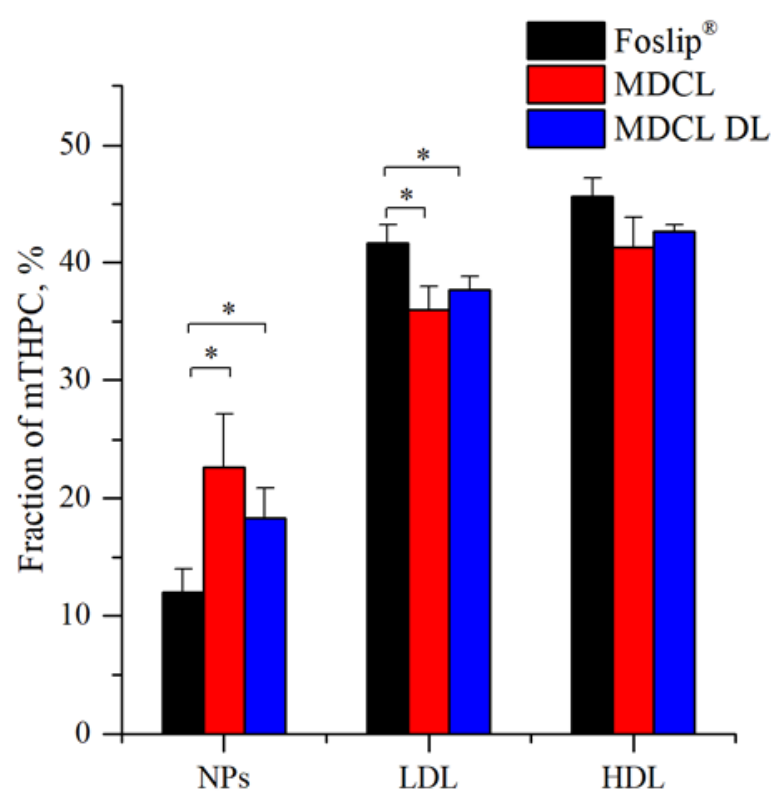

Figure 3. Quantitative analysis of mTHPC distribution in human serum after 24h incubation of mTHPC nanoformulations with $50 \%$ serum solution. * significantly different data points $(\mathrm{p}<0.05)$.

\subsection{Serum-depended cellular uptake}

To assess the influence of serum on the cellular accumulation of mTHPC, we performed flow cytometry analysis of HT29 monolayer cells after 24h incubation with Foslip ${ }^{\circledR}$ and MDCL DL in medium supplemented with various FBS concentration (Figure 4). The data presented as a mean mTHPC cellular fluorescence and uptake in serum-free medium was taken as $100 \%$. The cellular uptake of Foslip ${ }^{\circledR}$ strongly depended on the FBS concentration, that was already demonstrated on human biliary tract cancer cell lines ${ }^{25}$. The increase of serum concentration inhibits the accumulation of mTHPC in cells for both nanoformulations, however, in the case of MDCL DL the influence of serum was significantly less for FBS concentrations $>2 \%$. Thus, the results obtained confirmed better serum-stability of hybrid mTHPC/Me- $\beta$ CD-loaded liposomes.

\section{CONCLUSION}

Single and Double loaded hybrid DCL nanoconstructs have been prepared. We demonstrated higher serum-stability of hybrid liposomes compared with conventional mTHPC liposomal formulation (Foslip ${ }^{\circledR}$ ). In the case of DCLs, the amount of mTHPC remained in nanoparticles after 24h incubation in 50\% human serum was almost 2 times higher than that in Foslip ${ }^{\circledR}$. In fine, we demonstrated that the presence of serum in the culture medium less affected cellular uptake of 
mTHPC delivered by double loaded MDCL DL compared with Foslip ${ }^{\circledR}$. The data obtained confirm the interest of hybrid DCL nanoconstructs as a nanodelivery system for mTHPC.

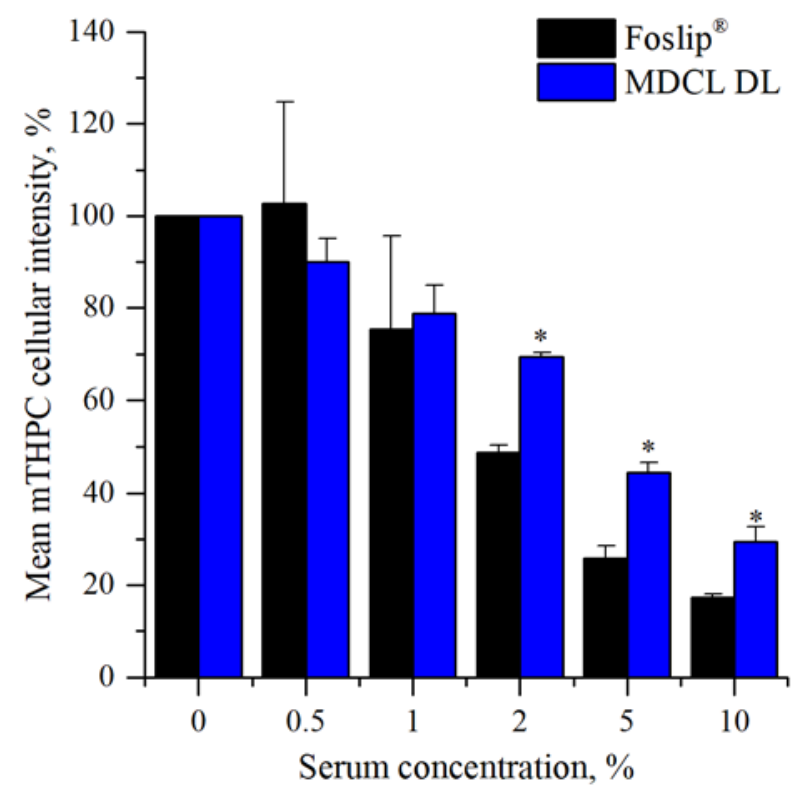

Figure 4. Serum-dependent mTHPC accumulation in HT29 monolayer cells. HT29 cells were incubated with Foslip ${ }^{\circledR}$ (black bars) and MDCL DL (blue bars) for 24h in serum-free medium or supplemented with 0.5, 1, 2, 5 and 10\% of FBS. The data presented as a mean mTHPC fluorescence of cells normalized to the uptake in serum-free medium. * indicates MDCL DL results significantly $(\mathrm{p}<0.05)$ different from the corresponding Foslip ${ }^{\circledR}$ data points.

\section{ACKNOWLEDGMENTS}

This work was supported by the Institut de Cancérologie de Lorraine, French "Ligue Nationale contre le Cancer (CCIRGE)”, Belarusian Republican Foundation for Fundamental Research (BRFFR) [grant numbers: M17MC-028, M18MB002] and the Ministry of Education of the Republic. The authors thank biolitec research GmbH (Jena, Germany) for providing with mTHPC. IY thanks Cancéropôle Est for the financial support for the participation at $17^{\text {th }}$ International Photodynamic Association World Congress (Boston, USA, 2019).

\section{REFERENCES}

[1] Dolmans, D. E. J. G. J., Fukumura, D. and Jain, R. K., “Photodynamic therapy for cancer,” Nature Reviews Cancer 3(5), 380-387 (2003).

[2] Bhuvaneswari, R., Gan, Y. Y., Soo, K. C. and Olivo, M., "The effect of photodynamic therapy on tumor angiogenesis,” Cell. Mol. Life Sci. 66(14), 2275-2283 (2009).

[3] Castano, A. P., Mroz, P. and Hamblin, M. R., "Photodynamic therapy and anti-tumour immunity," Nat. Rev. Cancer 6(7), 535-545 (2006).

[4] Marchal, S., Hor, A. E., Millard, M., Gillon, V. and Bezdetnaya, L., “Anticancer Drug Delivery: An Update on Clinically Applied Nanotherapeutics,” Drugs 75(14), 1601-1611 (2015).

[5] Senge, M. O. and Brandt, J. C., "Temoporfin (Foscan ${ }^{\circledR}$, 5,10,15,20-tetra(m-hydroxyphenyl)chlorin)--a secondgeneration photosensitizer," Photochem. Photobiol. 87(6), 1240-1296 (2011).

[6] Senge, M. O., "mTHPC - A drug on its way from second to third generation photosensitizer?," Photodiagnosis and Photodynamic Therapy 9(2), 170-179 (2012). 
[7] Senge, M. O. and Brandt, J. C., “Temoporfin (Foscan ${ }^{\circledR}$, 5,10,15,20-tetra(m-hydroxyphenyl)chlorin)--a secondgeneration photosensitizer,” Photochem. Photobiol. 87(6), 1240-1296 (2011).

[8] Yakavets, I., Millard, M., Zorin, V., Lassalle, H.-P. and Bezdetnaya, L., "Current state of the nanoscale delivery systems for temoporfin-based photodynamic therapy: Advanced delivery strategies,” J Control Release (2019).

[9] Yakavets, I., Lassalle, H.-P., Scheglmann, D., Wiehe, A., Zorin, V., Bezdetnaya, L., Yakavets, I., Lassalle, H.-P., Scheglmann, D., Wiehe, A., Zorin, V. and Bezdetnaya, L., "Temoporfin-in-Cyclodextrin-in-Liposome-A New Approach for Anticancer Drug Delivery: The Optimization of Composition,” Nanomaterials 8(10), 847 (2018).

[10] McCormack, B. and Gregoriadis, G., "Entrapment of cyclodextrin-drug complexes into liposomes: potential advantages in drug delivery,” J Drug Target 2(5), 449-454 (1994).

[11] Gharib, R., Greige-Gerges, H., Jraij, A., Auezova, L. and Charcosset, C., "Preparation of drug-in-cyclodextrinin-liposomes at a large scale using a membrane contactor: Application to trans-anethole,” Carbohydr Polym 154, 276-286 (2016).

[12] Dhule, S. S., Penfornis, P., Frazier, T., Walker, R., Feldman, J., Tan, G., He, J., Alb, A., John, V. and Pochampally, R., "Curcumin-loaded $\gamma$-cyclodextrin liposomal nanoparticles as delivery vehicles for osteosarcoma," Nanomedicine 8(4), 440-451 (2012).

[13] Yankovsky, I., Bastien, E., Yakavets, I., Khludeyev, I., Lassalle, H.-P., Gräfe, S., Bezdetnaya, L. and Zorin, V., "Inclusion complexation with $\beta$-cyclodextrin derivatives alters photodynamic activity and biodistribution of meta-tetra(hydroxyphenyl)chlorin,” Eur J Pharm Sci 91, 172-182 (2016).

[14] Yakavets, I., Yankovsky, I., Millard, M., Lamy, L., Lassalle, H.-P., Wiehe, A., Zorin, V. and Bezdetnaya, L., "The alteration of temoporfin distribution in multicellular tumor spheroids by $\beta$-cyclodextrins," Int J Pharm 529(1-2), 568-575 (2017).

[15] Reshetov, V., Lassalle, H.-P., François, A., Dumas, D., Hupont, S., Gräfe, S., Filipe, V., Jiskoot, W., Guillemin, F., Zorin, V. and Bezdetnaya, L., "Photodynamic therapy with conventional and PEGylated liposomal formulations of mTHPC (temoporfin): comparison of treatment efficacy and distribution characteristics in vivo,” Int J Nanomedicine 8, 3817-3831 (2013).

[16] Bonnett, R., Charlesworth, P., Djelal, B. D., Foley, S., McGarvey, D. J. and Truscott, T. G., "Photophysical properties of 5,10,15,20-tetrakis(m-hydroxyphenyl)porphyrin (m-THPP), 5,10,15,20-tetrakis(mhydroxyphenyl)chlorin (m-THPC) and 5,10,15,20-tetrakis(m-hydroxyphenyl)bacteriochlorin (m-THPBC): a comparative study,” J. Chem. Soc., Perkin Trans. 2(2), 325-328 (1999).

[17] Reshetov, V., Zorin, V., Siupa, A., D’Hallewin, M.-A., Guillemin, F. and Bezdetnaya, L., "Interaction of liposomal formulations of meta-tetra(hydroxyphenyl)chlorin (temoporfin) with serum proteins: protein binding and liposome destruction,” Photochem. Photobiol. 88(5), 1256-1264 (2012).

[18] Torchilin, V. and Weissig, V., eds., [Liposomes: A Practical Approach, Second Edition], Oxford University Press, Oxford, New York (2003).

[19] Yakavets, I. V., Yankovsky, I. V., Khludeyev, I. I., Lassalle, H. P., Bezdetnaya, L. N. and Zorin, V. P., “Optical Methods for the Analysis of the Temoprofin Photosensitizer Distribution Between Serum Proteins and Methyl- $\beta$ Cyclodextrin Nanocarriers in Blood Serum,” J Appl Spectrosc 84(6), 1030-1036 (2018).

[20] Yakavets, I., Yankovsky, I., Bezdetnaya, L. and Zorin, V., "Soret band shape indicates mTHPC distribution between $\beta$-cyclodextrins and serum proteins,” Dyes and Pigments 137, 299-306 (2017).

[21] Millard, M., Yakavets, I., Piffoux, M., Brun, A., Gazeau, F., Guigner, J.-M., Jasniewski, J., Lassalle, H.-P., Wilhelm, C. and Bezdetnaya, L., "mTHPC-loaded extracellular vesicles outperform liposomal and free mTHPC formulations by an increased stability, drug delivery efficiency and cytotoxic effect in tridimensional model of tumors,” Drug Delivery 25(1), 1790-1801 (2018).

[22] Bonté, F. and Juliano, R. L., "Interactions of liposomes with serum proteins," Chemistry and Physics of Lipids 40(2), 359-372 (1986).

[23] Chen, H., Gao, J., Wang, F. and Liang, W., "Preparation, Characterization and Pharmacokinetics of LiposomesEncapsulated Cyclodextrins Inclusion Complexes for Hydrophobic Drugs,” Drug Delivery 14(4), 201-208 (2007).

[24] Maestrelli, F., González-Rodríguez, M. L., Rabasco, A. M. and Mura, P., "Effect of preparation technique on the properties of liposomes encapsulating ketoprofen-cyclodextrin complexes aimed for transdermal delivery,” Int J Pharm 312(1-2), 53-60 (2006).

[25] Kiesslich, T., Berlanda, J., Plaetzer, K., Krammer, B. and Berr, F., “Comparative characterization of the efficiency and cellular pharmacokinetics of Foscan- and Foslip-based photodynamic treatment in human biliary tract cancer cell lines," Photochem. Photobiol. Sci. 6(6), 619-627 (2007). 